\title{
Evaluación multicriterio para la optimización de redes de energía
}

\author{
Ascensión Zafra, Rafael Espinosa, Miguel Ángel Ridao, Carlos Bordóns \\ Departamento de Ingeniería de Sistemas y Automática. \\ Escuela Técnica Superior de Ingeniería \\ Universidad de Sevilla \\ \{asunzafra,bordons,miguelridao\}@us.es, rafaelespinosa2005@hotmail.com
}

\section{Resumen}

Este trabajo propone una herramienta para ayudar en la toma de decisión de la planificación de redes de energía. Se incluye una evaluación multicriterio de los escenarios posibles de planificación considerando distintos criterios y ponderaciones. Para este cometido se usa la herramienta multiobjetivo discreta PROMETHEE y planos GAIA. El conjunto de escenarios se genera considerando incertidumbres que puede presentar el sistema. La simulación de la red eléctrica se lleva a cabo en la herramienta comercial OpenDSS. Para ilustrar la herramienta se ha tomado una red de la IEEE, donde se observan los beneficios del método propuesto. Los resultados obtenidos muestran que el tomar incertidumbres en el proceso de optimización de las redes de potencia, supone un gran aumento en la eficiencia de la red.

Palabras clave: Gestión Microrredes, Optimización Multicriterio, Incertidumbres, Simulación

\section{INTRODUCCIÓN}

Estamos en una era donde se está realizando una transición del sistema eléctrico tradicional a redes de menor tamaño con incorporación de sistemas de almacenamiento. Las previsiones de energía indican un crecimiento moderado de la demanda, un fuerte incremento de las energías renovables y una necesidad de potencia firme y flexible. Todo ello está haciendo que el concepto de red eléctrica "inteligente" y microrredes estén tomando un considerable auge, propiciando el desarrollo de técnicas de gestión que mejoren el rendimiento y desempeño de la red [5].

Las redes eléctricas inteligentes (o smart grids en inglés) integran las tecnologías de la información con las infraestructuras eléctricas actuales, haciendo posible una comunicación bidireccional entre generadores, y consumidores. El nuevo modelo energético pretende transformar el sistema actual en un sistema distribuido, en el cual, cualquier agente que esté conectado a la red tiene la posibi- lidad de aportar energía, apoyando la creación de microgeneradores. De esta forma, no existe una dependencia tan directa como con la generación energética actual. Las redes inteligentes disminuyen drásticamente las pérdidas por el transporte energético, facilitan la conexión a la red de todo tipo de energías renovables (mejorando la integración de porcentajes crecientes de energías no gestionables como la eólica o la solar), soportan las capacidades de almacenamiento energético y la conexión masiva de vehículos eléctricos o híbridos (tanto para cargar como para volcar energía a la red)[12].

Dentro de las redes eléctricas inteligentes, se pueden distinguir las microrredes, inicialmente introducidas en [11]. Se trata de una alternativa para abordar la integración de recursos energéticos distribuidos, como las energías renovables o los coches eléctricos. Según [7], las microrredes pueden considerarse como un conjunto de cargas, generadores y sistemas de almacenamiento coordinadas tal que puedan suministrar electricidad de forma factible. Pueden funcionar de forma aislada o conectadas a la red principal, vistas desde esta última como un elemento único que responde a las señales de control. Es por tanto necesario controlar la microrred para que desempeñe su función de forma estable y económica.

Usualmente, en el control de las microrredes se plantean problemas de optimización en los cuales se valoran distintos objetivos $[8,6]$. Así por ejemplo, en la planificación diaria de una microrred se ha de valorar la satisfacción de las demandas, el coste económico, la fiabilidad de los componentes,... llegando a una solución de compromiso entre el conjunto de factores. La mejor estrategia a seguir dependerá en parte de los objetivos considerados, sus prioridades y ponderaciones.

Se pueden encontrar en la literatura, distintas técnicas de decisión multicriterio (o multiobjetivo) aplicadas a sistemas ([1], [10]). Una posible clasificación de estos métodos se puede hacer atendiendo si la región factible del problema es continua o discreta. Dentro de esta última se destaca la técnica PROMETHEE (Preference Ranking Organisational Method for Enrichment Evaluation) 


\section{[4].}

Este trabajo presenta una herramienta de decisión multiobjetivo para la optimización de la planificación de microrredes. La generación de las distintas alternativas de configuración de la microrred se realiza considerando incertidumbres. En este trabajo la fuente de incertidumbres viene determinada por la incorporación de fuentes de energía renovables y coches eléctricos en la microrred. De esta forma, el conjunto de alternativas y por tanto las posibilidades del decisor se ve incrementada. La simulación de cada alternativa se lleva a cabo en el paquete software OpenDSS, donde se valoran las pérdidas de la red en cada uno de los nodos y la satisfacción de la demanda. Los resultados de la simulación son usadas en la evaluación multicriterio usando la técnica PROMETHEE y planos GAIA (Geometrical Analysis for Interactive Assistance), obteniendo la mejor de las opciones de la configuración de la microrred ([3]).

La organización del artículo es la siguiente. La sección 2 expone cómo se lleva a cabo la generación de alternativas de configuración de una red de energía. La sección 3 describe cómo se evalúan cada una de estas alternativas, considerando indicadores como pérdidas, satisfacción de la demanda, etc... En este apartado se describe la herramienta de simulación OPenDSS. La evaluación multicriterio se describe en la sección 4, con la técnica PROMETHEE y planos GAIA. En la sección 5 se usa la microrred de la IEEE de 37 nodos a la cual se le han realizado algunos cambios para incluir coches eléctricos y otras fuentes renovables, poniendo de manifiesto cada uno de los pasos seguidos. Finalmente, el capítulo 6 concluye este artículo, mostrando las conclusiones.

\section{GENERACIÓN DE ESCENARIOS DE CONFIGURACIÓN DE MICRORRED}

Este punto tiene como objetivo generar un conjunto de escenarios posibles de configuración de la microrred con objeto de elegir la más idónea atendiendo a una evaluación multicriterio.

Para ello, se van a introducir variabilidad en las siguientes variables:

- Costes de producir cada unidad de potencia de las fuentes, $C_{i}$ (euros)

- Cargas puntuales en cada uno de los nodos de la red, considerando dentro de ellas las conexiones de los coches eléctricos, $L_{i}(\mathrm{kWh})$
- Potencia suministrada por cada uno de los generadores de la microrred, $P_{i}(\mathrm{kWh})$

Partiendo de una configuración inicial, al que llamaremos escenario nominal, se generarán las distintas realizaciones de estas variables atendiendo a variables estocásticas. En nuestro caso, se modelan con funciones de distribución de probabilidad identificando datos históricos. Las funciones de distribución se discretizarán en un conjunto finito de escenarios posibles [2].

Un proceso estocástico, $\mathbf{P}$, se define de forma generalizada como una colección de $n a$ variables aleatorias dependientes:

$$
\mathbf{P}=\left\{y_{t}^{i}, t \in T, i \in\{1 \ldots n a\}\right\},
$$

donde para cada $t$ en el espacio temporal $T, y_{t}^{i}$ es el estado de la variable aleatoria $i$ en el tiempo $t$. Se trata ahora de determinar la estructura probabilística de las variables $\left\{y_{t}^{i}\right\}$, estudiando su comportamiento probabilístico y la interrelación entre ellas (dependencia estática). Este procedimiento puede ser bastante complejo; en nuestro caso se asume que el proceso estocástico sigue una distribución Gaussiana en la que se ha de especificar el vector de valores medios y la matriz de covarianza. En [13] se puede ver cómo modelar un proceso estocástico si no se asume lo anterior y se recurre a series temporales basadas en modelos ARMA (AutoRegressive Moving Average).

\section{SIMULACIÓN DE ALTERNATIVAS}

Considérese el conjunto de $n$ alternativas $\left\{A_{1}, A_{2}, \ldots, A_{n}\right\}$ y $m$ criterios de evaluación. En este apartado se trata de evaluar cada alternativa respecto a cada criterio, $f^{c}\left(A_{i}\right)$, con $c=1 \ldots m$ y $i=1 \ldots n$. En nuestro caso, las distintas alternativas son parámetros de configuración de la red de energía. Para obtener los resultados se necesita el modelo de la red. En este trabajo se va a usar para la simulación el software OpenDSS (Open-source Distribution System Simulator) [9].

OpenDSS es un software de simulación de sistemas de distribución de energía eléctrica, desarrollado por EPRI (Electric Power Research Institute). Es un programa de simulación en el dominio de la frecuencia con las funcionalidades propias de los simuladores comerciales, así como nuevas herramientas que tienen en cuenta futuras necesidades relacionadas con los esfuerzos de modernización de las redes actuales. El programa fue originalmente pensado como una herramienta para el análisis de la interconexión de generación distribuida, pero su continua evolución ha llevado al 
desarrollo de otras funcionalidades que son adecuadas, por ejemplo, para estudios de eficiencia en el suministro de energía y estudios de armónicos. OpenDSS representa los circuitos de distribución a través de ecuaciones de admitancias nodales. Cada elemento del sistema es representado por una matriz de admitancia nodal primitiva. Cada matriz primitiva es adjuntada a la matriz de admitancias del sistema, por lo que el sistema de ecuaciones que representa al sistema es solucionado con la ayuda de algoritmos de matrices dispersas. OpenDSS está diseñado para recibir instrucciones y devolver resultados en formato de texto. Puede accederse al programa tanto a través de una aplicación independiente como del módulo COM server. La aplicación independiente cuenta con una interfaz de usuario básica, aunque completamente funcional, que permite interactuar con el programa, mientras que el COM server permite conectar el OpenDSS con otros programas tales como Matlab, proporcionando de esa manera una gran capacidad de análisis de la información. La figura 1 muestra cómo interactúan los diferentes módulos dentro de la estructura de OpenDSS.

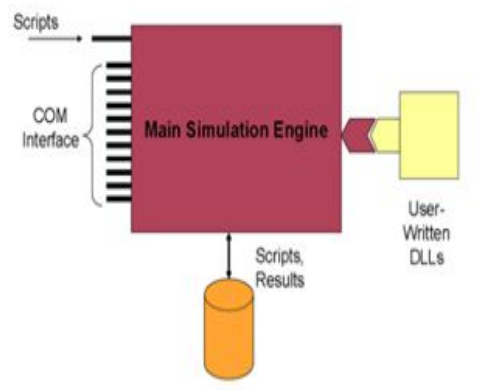

Figura 1: Esquema OpenDSS

En este trabajo las distintas alternativas se ejecutan desde un módulo de programación en Matlab.

\section{EVALUACIÓN MULTICRITERIO}

Para la evaluación de las distintas alternativas de configuración de la red, se ha optado por el método discreto PROMETHEE [4]. Esta técnica parte de la base de que el decisor debe establecer la importancia relativa de cada uno de los criterios para luego definir una estructura de preferencia entre las alternativas. De esta forma, se definen funciones de preferencia que evitan la ambigüedad en la determinación de los umbrales de cada criterio. Para cada criterio se asocia una función con la que se valora la preferencia de una alternativa respecto a otra. De esta forma $P_{c}\left(A_{i}, A_{k}\right)$ representa la decisión entre la alternativa $A_{i}$ y $A_{k}$ con respecto al criterio $c$. El valor obtenido está en el rango entre
0 y 1.

Para aplicar el método PROMETHEE se necesita de forma adicional los pesos de cada criterio, $w_{j}$. Se computa para cada par de alternativas $A_{i}$ y $A_{k}$ la decisión con respecto a todos los criterios, expresada de la forma:

$$
\pi\left(A_{i}, A_{k}\right)=\frac{\sum_{j=1}^{m} P_{j}\left(A_{i}, A_{k}\right)}{\sum_{j=1}^{m} w_{j}}
$$

Con el fin de posicionar cada alternativa con respecto a las demás, PROMETHEE computa los flujos de preferencia positivo y negativo, $\phi^{+}$y $\phi^{-}$ para cada alternativa.

$$
\begin{aligned}
& \phi^{+}\left(A_{i}\right)=\frac{1}{n-1} \sum_{k=1}^{n} \pi\left(A_{i}, A_{k}\right) \\
& \phi^{-}\left(A_{i}\right)=\frac{1}{n-1} \sum_{k=1}^{n} \pi\left(A_{k}, A_{i}\right)
\end{aligned}
$$

El flujo positivo $\phi^{+}\left(A_{i}\right)$ cuantifica cómo la alternativa $A_{i}$ es dominante sobre las otras. El flujo negativo $\phi^{-}\left(A_{i}\right)$ representa cuánto es dominado por las otras alternativas. La diferencia de ambos, el flujo neto $\phi$, se obtiene para ordenar las alternativas:

$$
\phi\left(A_{i}\right)=\phi^{+}\left(A_{i}\right)-\phi^{-}\left(A_{i}\right)
$$

Un valor mayor en una alternativa que en otra de esta variable, representa una mejor valoración.

\section{$5 \quad$ EJEMPLO: RED IEEE DE 37 NODOS AMPLIADA}

La red de distribución que se usará para ilustrar el método corresponde al Test CaseIEEE 37-bus Feeder, compuesta de 37 nodos. En esta red se puede diferenciar un regulador entre el bus $799 \mathrm{y}$ el nodo 701 y un transformador entre los nodos 709 y 775. Como ya se ha descrito con anterioridad, se utilizará una modificación de esta red de distribución. La principal diferencia es la incorporación de diferentes agentes a esta red, tales como una batería (o grupo electrógeno), una pila de combustible, un generador solar, un generador eólico y un coche eléctrico. Los nodos donde colocaremos estos agentes serán los nodos 701, 720, 730,722 y 737 respectivamente. La figura 2 muestra la configuración de esta red. Las propiedades de cada uno de los nodos se describen en un fichero de texto que toma como entrada de datos el simulador.

\subsection{PROBLEMA DE OPTIMIZACIÓN}

El objetivo principal de nuestro problema será el de satisfacer la demanda de energía de nuestra red 


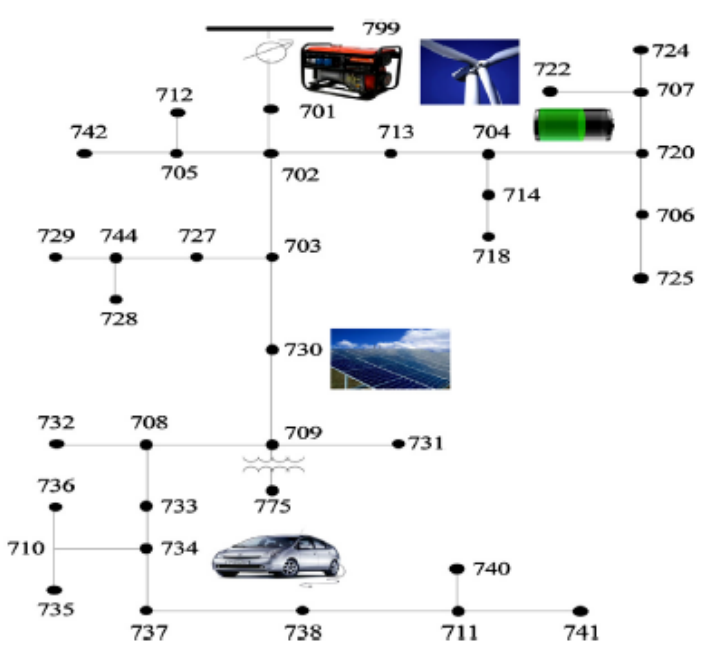

Figura 2: Red de energía de ejemplo.

y de los coches eléctricos (mediante el empleo de los diferentes generadores ya implementados en OpenDSS), reducir los costes de operación y reducir en todo lo posible las pérdidas que se puedan producir en la red. Esto queda reflejado en la siguiente función objetivo:

$$
\begin{aligned}
F & =\beta_{1} \sum_{t=1}^{24}(D(t)-E(t))^{2}+ \\
& +\beta_{2} \sum_{t=1}^{24} \sum_{j=1}^{4}\left(C_{j}(t) * P_{j}(t)+\right. \\
& +\beta_{3} \sum_{t=1}^{24} P_{e r}(t)
\end{aligned}
$$

donde $D(t)$ es la suma de la demanda de energía de los $n_{i}$ coches eléctricos y de la red; $E(t)$ es la suma de las energías producidas por los generadores, $C_{j}$ es el coste de producir una unidad de energía en el generador $j$ y $P_{j}$ es la potencia generada por el generador $j$. El término $P_{e r}(t)$ representa las pérdidas de potencia de la red por hora. Nótese que todos los sumatorios están realizados en un intervalo de 24 horas. El sumatorio $j=1 \ldots 4$, corresponde a los 4 generadores existentes en la red.

Los diferentes pesos de la función objetivo se han asignado como $\beta_{1}=0.4, \beta_{2}=0.4$ y $\beta_{3}=0.2$. Los valores de carga en cada uno de los nodos se establecen en el fichero de texto de entrada al simulador. Una vez calculadas las pérdidad de la red así como la comprobación del abastecimiento de los nodos, se pueden extraer los valores desde el entorno Matlab con los siguientes comandos:

DSSText.command='show generators';
DSSText.command='show meters'; DSSText.command='show losses';

El coste de producir el $\mathrm{kWh}$ para cada uno de los generadores, se han tomado de la web de IRENA (Agencia Internacional de Energías Renovables). Para las energías solares y eólicas, el rango de coste está entre 0.05 y $0.08 € / \mathrm{kWh}$. Para los combustibles fósiles y otras energías entre 0.045 y $0.14 € / \mathrm{kWh}$. La tabla 1 muestra los valores nominales de coste por $\mathrm{kWh}$ y potencia nominal de los 4 generadores.

Tabla 1: Valores nominales de costes y potencias de los generadores.

\begin{tabular}{|l|l|l|}
\hline Generador & Coste $(€)$ & Potencia \\
\hline G. Electrógeno & $C_{1}=0.045$ & $P_{1}=1 \mathrm{~kW}$ \\
P. de Combustible & $C_{2}=0.05$ & $P_{2}=8 \mathrm{~kW}$ \\
Energía eólica & $C_{3}=0.05$ & $P_{3}=2300 \mathrm{~kW}$ \\
Energía solar & $C_{4}=0.08$ & $P_{4}=78 \mathrm{~kW}$ \\
\hline
\end{tabular}

La decisión a tomar en este problema viene determinada por las potencias de entrada de los generadores.

Para la generación del conjunto de alternativas, según se establece en la sección 2 , se han considerado como variables estocásticas los siguientes valores:

- Los costes de producir cada unidad de potencia , $\left\{C_{1}, C_{2}, C_{3}, C_{4}\right\}$.

- Valor de las cargas de los coches eléctricos dependiendo del instante de tiempo, $L_{i}$.

- Potencia suministrada por los generadores $\left(\left\{P_{1}, P_{2}, P_{3}, P_{4}\right\}\right)$.

\subsection{RESULTADOS}

Cada una de las alternativas propuestas se ha evaluado atendiendo a los 3 criterios establecidos en la expresión 5.1 y obteniendo los resultados del software de simulación OpenDSS. El siguiente paso es introducir cada una de las alternativas y su valoración con respecto a cada criterio en el programa Visual Promethee-GAIA.

Los experimentos realizados se han clasificado dependiendo de la generación de alternativas. Se verá a continuación que los primeros escenarios se generan cambiando sólo un criterio en cada alternativa. En el segundo bloque, las alternativas se generan modificando varios criterios en cada alternativa. 


\subsubsection{Alternativas con incertidumbres en un sólo criterio}

En este primer grupo de resultados, las alternativas se han generado introduciendo incertidumbres en un sólo criterio. Para el resto de criterios, permanecen los valores nominales. De esta forma se han llevado a cabo 3 grupos de evaluaciones. En la primera evaluación, las alternativas sólo han visto modificada la potencia de los generadores; en la segunda, las cargas de los nodos y en la tercera, los costes de producción de la energía. A título de ejemplo, la figura 3 muestra los resultados de PROMETHEE cuando se evalúan 7 alternativas correspondientes a variar los costes de producción de energía. El resultado que devuelve establece que la mejor alternativa es la $A_{19}$, consistente en aumentar el coste del $\mathrm{kW}$ del grupo electrógeno en un 20\%. Las 3 columnas últimas muestran el flujo neto, flujo positivo y flujo negativo. Nótese que las alternativas se ordenan atendiendo al flujo neto.

\begin{tabular}{|c|l|r|rrr|}
\hline Rank & \multicolumn{2}{|c|}{ action } & Phi & Phi+ & Phi- \\
$\mathbf{1}$ & Alternativa 19 & $\square$ & 0,4571 & 0,4571 & 0,0000 \\
$\mathbf{2}$ & Alternativa 15 & $\square$ & 0,3429 & 0,4000 & 0,0571 \\
$\mathbf{3}$ & Alternativa 16 & $\square$ & 0,2286 & 0,3429 & 0,1143 \\
$\mathbf{4}$ & Alternativa 20 & $\square$ & 0,1143 & 0,2857 & 0,1714 \\
$\mathbf{5}$ & Alternativa 22 & $\square$ & $-0,0571$ & 0,1714 & 0,2286 \\
$\mathbf{6}$ & Alternativa 17 & $\square$ & $-0,1143$ & 0,1714 & 0,2857 \\
$\mathbf{7}$ & Alternativa 18 & $\square$ & $-0,2286$ & 0,1143 & 0,3429 \\
$\mathbf{8}$ & Alternativa 21 & $\square$ & $-0,7429$ & 0,0000 & 0,7429 \\
\hline
\end{tabular}

Figura 3: Evaluación multicriterio de alternativas con cambios en coste del Kw.

En la siguiente simulación, se comparan las 3 alternativas mejores resultantes de variar potencias, cargas y costes de forma independiente, como se ha detallado anteriormente, siendo éstas:

- $A_{5}$ : Se mantienen cargas y costes y se reduce potencia nominal en un $10 \%$.

- $A_{8}$ : Se mantienen potencias nominales y costes y se reducem cargas en un $5 \%$.

- $A_{19}$ : Se mantienen potencias nominales y cargas y se aumenta el coste del grupo eléctrogeno en un $20 \%$.

De la figura 4 se puede deducir que si sólo se produjesen cambios en una de las tres direcciones o criterios que se han establecido, la mejor opción sería mantener los costes y las potencias nominales iniciales, intentando reducir las cargas $\left(A_{8}\right.$, cargas al 95\%), es decir, que el consumo sea menor. Esto ajustaría al máximo la diferencia entre demanda y generación de potencia.

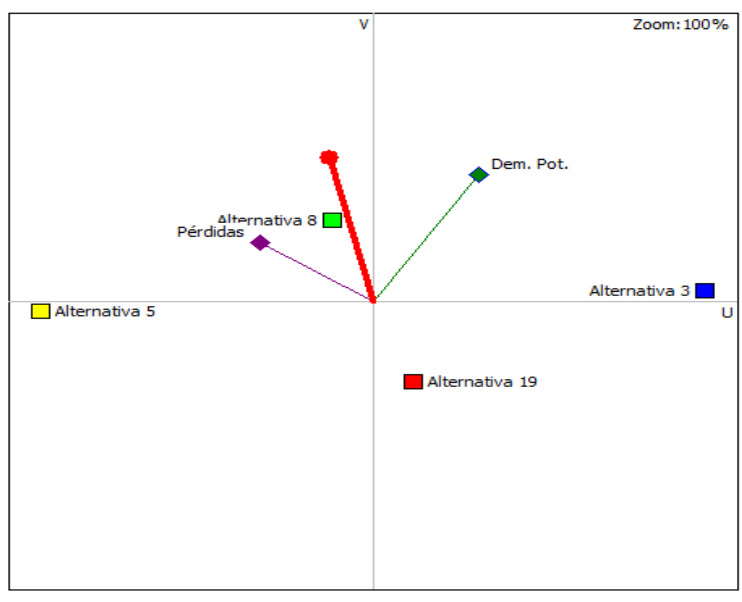

Figura 4: Plano GAIA.

Se puede observar en el plano GAIA dónde se encontraría la localización de la mejor alternativa. La $A_{8}$ es la más cercana a esta dirección de mejora. Sin embargo la alternativa $A_{19}$ está justo en la dirección contraria a ella. Sería interesante ver, no sólo poder comparar situaciones con valores fijos, si no con rangos de valores. Para esto tenemos la curva de eficiencia o Frente de Pareto, donde observamos por donde nos podemos mover eficientemente al aumentar o disminuir el coste o la satisfación de la demanda (ver figura 5).

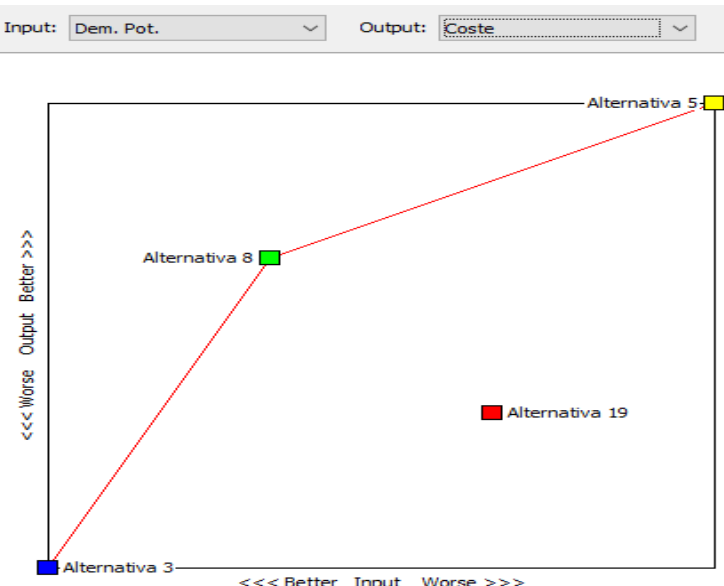

Figura 5: Frente Pareto Demanda- Coste.

La siguiente simulación pone de manifiesto como afecta la decisión cuando se cambian los pesos en la función objetivo. En este caso se han cambiado los pesos a $\beta_{1}=0.25, \beta_{2}=0.5$ y $\beta_{3}=0.25$. Como se puede observar en la figura 6 la mejor alternativa ahora cambia, siendo la alternativa $A_{5}$.

\subsubsection{Alternativas con incertidumbres en varios criterios}

Todas las decisiones tomadas en el apartado anterior ponen de manifiesto cómo actuar si sólo se 

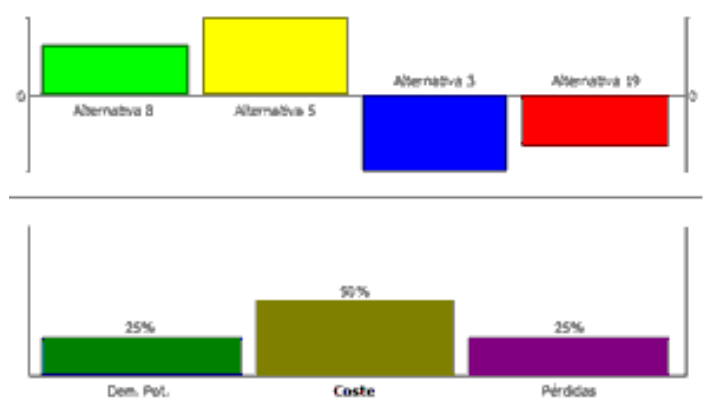

Figura 6: Evaluación de alternativas con cambio en los pesos de la función objetivo.

presentan cambios en una dirección. Este escenario es poco probable, por lo que el estudio de cambios en varios criterios parece la mejor forma de comparar los resultados.

La tabla 5.2.2 presenta las alternativas para las modificaciones combinadas, realizadas a partir de la situación inicial. Así por ejemplo, la alternativa $A_{3}$ considera un incremento en la demanda del $10 \%$ y una reducción en la producción de energía del $10 \%$.

Tabla 2: Generación de alternativas modificando varios criterios.

\begin{tabular}{|l|l|l|l|}
\hline Alternativa & Carga & Potencia & Coste \\
\hline$A_{1}$ & - & - & - \\
$A_{2}$ & 105 & 95 & - \\
$A_{3}$ & 110 & 90 & - \\
$A_{4}$ & 95 & 105 & - \\
$A_{5}$ & 90 & 110 & - \\
$A_{6}$ & 105 & - & 95 \\
$A_{7}$ & 110 & - & 90 \\
$A_{8}$ & 95 & - & 105 \\
$A_{9}$ & 90 & - & 110 \\
$A_{10}$ & - & 105 & 95 \\
$A_{11}$ & - & 110 & 90 \\
$A_{12}$ & - & 95 & 105 \\
$A_{13}$ & - & 90 & 110 \\
$A_{14}$ & 110 & 90 & 110 \\
$A_{15}$ & 110 & 110 & 110 \\
$A_{16}$ & 90 & 90 & 110 \\
$A_{17}$ & 90 & 110 & 110 \\
$A_{18}$ & 110 & 110 & 90 \\
$A_{19}$ & 110 & 90 & 90 \\
$A_{20}$ & 90 & 110 & 90 \\
$A_{21}$ & 90 & 90 & 90 \\
\hline
\end{tabular}

Los resultados obtenidos con PROMETHEE se muestran en la figura 7 . Se pueden deducir los siguientes comentarios:

- La situación más favorable es reducir las potencias de los generadores si se detecta que se satisface correctamente la demanda y se ha producido una reducción de costes $\left(A_{21}\right)$. La alternativa $A_{16}$ obtiene un resultado parecido, aumentando el coste en un $10 \%$.

- Si se produjese una reducción de costes, se podría aumentar la potencia generada en un $5 \%\left(A_{10}\right)$ para obtener un buen resultado. Si esto no es posible, se podría permitir un aumento en las cargas de un $5 \%\left(A_{6}\right)$. Se empeoraría la satisfacción de la demanda pero se reducirían costes.

- Hay una gran cantidad de alternativas o situaciones que resultan con peor comportamiento a la inicial. Es el caso de todas aquellas que se encuentran por debajo de la alternativa $A_{1}$, presentando un flujo negativo. Muchas de ellas $\left(A_{5}, A_{17}\right.$ o $\left.A_{20}\right)$ se producen cuando se decide aumentar la producción de energía significativamente si se reducen las cargas, algo que claramente no se debe hacer.

\begin{tabular}{|c|c|c|c|c|}
\hline Rank & action & Phi & Phit & Phi- \\
\hline 1 & Alternativa 21 & 0,8700 & 0,9100 & 0,0400 \\
\hline 2 & Alternativa 16 & 0,5500 & 0,7100 & 0,1600 \\
\hline 3 & Alternativa 10 & 0,2200 & 0,6000 & 0,3300 \\
\hline 4 & Alternativa 6 & 0,2000 & 0,5900 & 0,3900 \\
\hline 5 & Alternativa 8 & 0,1600 & 0,5700 & 0,4100 \\
\hline 6 & Alternativa 11 & 0,1200 & 0,5100 & 0,3900 \\
\hline 7 & Alternativa 13 & 0,1200 & 0,5100 & 0,3900 \\
\hline 8 & Alternativa 19 & 0,1000 & 0,5100 & 0,4100 \\
\hline 9 & Altemativa 2 & 0,0600 & 0,5200 & 0,4600 \\
\hline 10 & Alternativa 7 & 0,0200 & 0,5000 & 0,4800 \\
\hline 11 & Alternativa 12 & 0,0200 & 0,5000 & 0,4800 \\
\hline 12 & Alternativa 3 & 0,0200 & 0,4700 & 0,4500 \\
\hline 13 & Alternativa 1 & $-0,0400$ & 0,4000 & 0,5200 \\
\hline 14 & Alternativa 4 & $-0,0400$ & 0,4700 & 0,5100 \\
\hline 15 & Alternativa 9 & $-0,0600$ & 0,4600 & 0,5200 \\
\hline 16 & Alternativa 18 & $-0,0700$ & 0,4000 & 0,4700 \\
\hline 17 & Alternativa 14 & $-0,2200$ & 0,3100 & 0,5300 \\
\hline 18 & Alternativa 20 & $=0,2400$ & 0,3000 & 0,5400 \\
\hline 19 & Alternativa 15 & $-0,5100$ & 0,2200 & 0,7300 \\
\hline 20 & Alternativa 5 & $-0,6000$ & 0,1600 & 0,7600 \\
\hline 21 & Alternativa 17 & $-0,6800$ & 0,1200 & 0,8000 \\
\hline
\end{tabular}

Figura 7: Evaluación de alternativas con cambios en varios criterios.

Las figuras 8 y 9 representan el Frente Pareto para el criterio satisfacción de demanda frente a las pérdidas de la red y costes de producción, respectivamente. Se puede observar que las alternativas $A_{21}$ y $A_{10}$ se encuentran en la optimimalidad para las dos comparaciones. Otras alternativas, dependiendo de la prioridad de la función objetivo, cambian su posición.

Para finalizar, se muestra el plano GAIA de todas las alternativas. Se observan las direcciones 

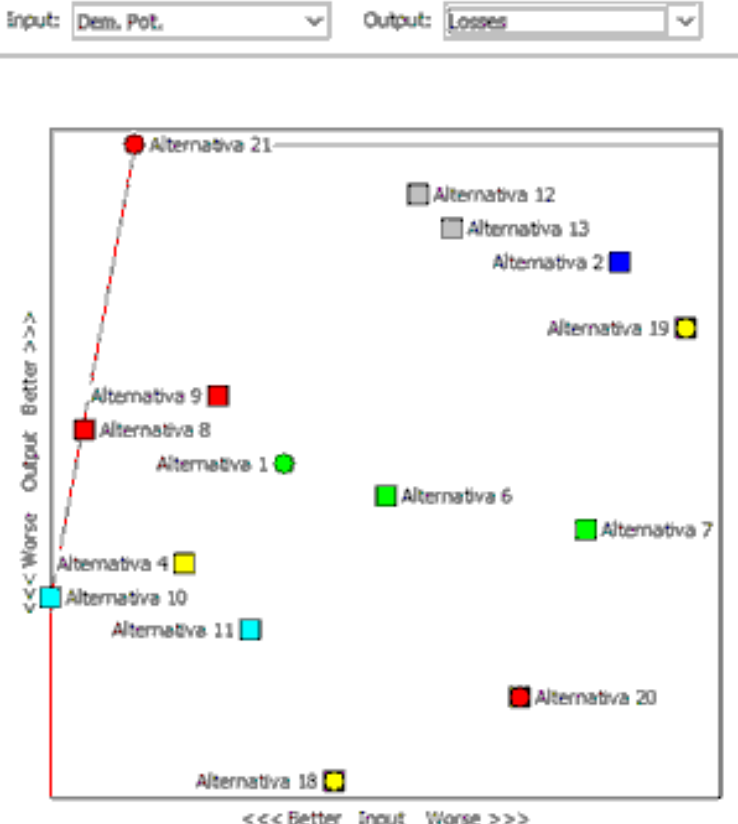

Figura 8: Frente Pareto Demanda-Pérdidas.
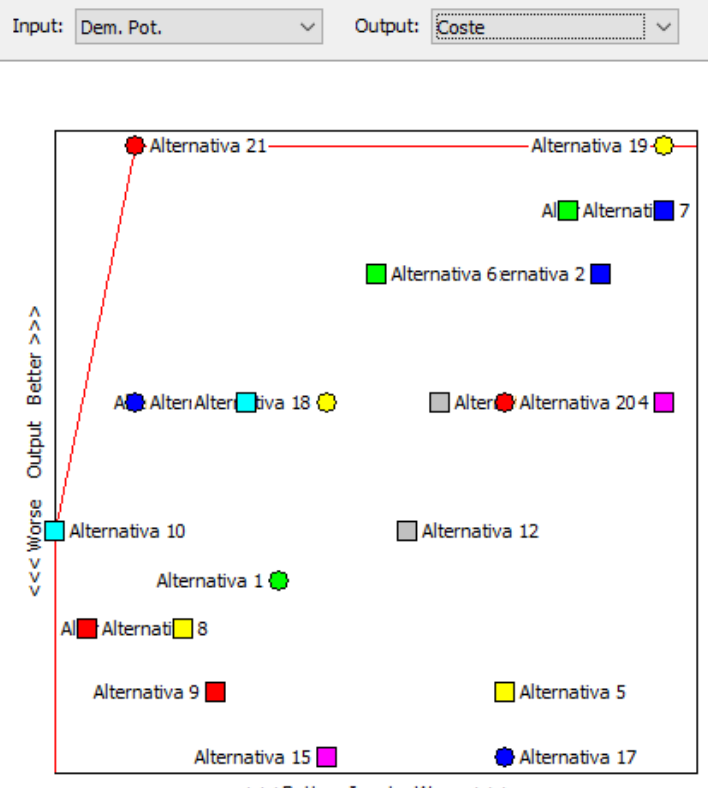

Figura 9: Frente Pareto Demanda-Costes.

de mejora de cada criterio y la combinación de los tres. Alternativas como la $A_{20}, A_{5}$ o $A_{15}$ se encuentran en sentido opuesto a la óptima, mientras que las alternativas $A_{21}, A_{12}$ o $A_{16}$ están en dirección y sentido hacia la mejor situación. Otras como la $A_{8}$ se encuentran opuestas al coste, pero muy favorables a la satisfacción de la demanda. De igual forma ocurre para la $A_{6}$ en relación al coste-demanda.

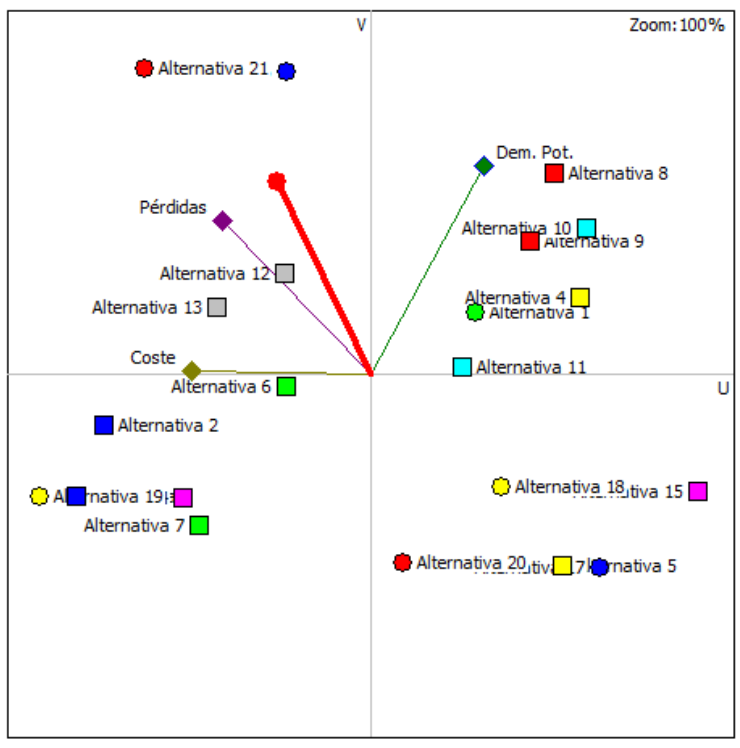

Figura 10: Plano GAIA.

\section{CONCLUSIONES}

La utilización del método de evaluación Promethee ha permitido optimizar varios criterios de forma simultánea presentados de una forma gráfica, ayudando a la toma de decisión. La incorporación de incertidumbres en las alternativas a valorar hace que la solución a determinar sea más eficiente.

La selección de OpenDSS como herramienta de simulación presenta ventajas evidentes; permite modelar cualquier tipo de red de energía con cargas constantes en los nodos o bien que varíen con el tiempo (diarias). Ello proporciona un valor agregado al contemplar condiciones más reales para el sistema.

\section{Agradecimientos}

Este trabajo ha sido realizado en el marco del proyecto CONFIGURA (DPI2016-78338-R) perteneciente al programa de proyectos de investigación del Ministerio de Economía, Industria y Competitividad de España.

\section{Referencias}

[1] C. Araz and I. Ozkarahan. Supplier evaluation and management system for strategic sourcing based on a new multicriteria sorting procedure. International Journal of Production Economics, 106(2):585-606, 2007.

[2] J.R. Birge and F. Louveaux. Introduction to stochastic programming. Springer-Verlag, 1997. 
[3] J. Brans and B. Mareschal. Promcalc \& gaia: a new decision support system for multicriteria decision aid. Decision Support Systems, $12,1994$.

[4] J.P. Brans and Ph Vincke. A preference ranking organisation method: The promethee method for mcdm. Management Science, 31(6):647-656, 1985.

[5] F. García-Torres C. Bordons and Luis Valverde. Gestión óptima de la energía en microrredes con generación renovable. Revista Iberoamericana de Automática e Informática Industrial, 12:117-132, 2015.

[6] N. Meskens D. Duvivier and M. Ahues. A fast multicriteria decision-making tool for industrial scheduling problems. Int. J. Production Economics, 145:753-760, 2013.

[7] A. H. Etemadi C. A. Canizares R. Iravani M. Kazerani A. H. Hajimiragha O. GomisBellmunt M. Saeedifard R. Palma-Behnke G. A. Jiménez-Estévez D. E. Olivares, A. Mehrizi-Sani and N.D. Hatziargyriou. Trends in microgrid control. IEEE Transactions on Smart Grid, 5(4):1905-1919, 2014.

[8] M. Esmaili and A. Goldoust. Multi-objective optimal charging of plug-in electric vehicles in unbalanced distribution networks. Electrical Power and Energy Systems, 73:644-652, 2015.

[9] Electric Power Research Institute. Opensource distribution system simulator (opendss).

[10] J. Jozefowska and A. Zimniak. Optimization tool for short-term production planning and scheduling. International Journal of Production Economics, 112(1):109-120, 2008.

[11] B. Lasseter. Microgrids (distributed power generation). In Proc. IEEE Power Engineer. Soc. Winter Meet, 1:146-149, 2001.

[12] Turismo y Agenda Digital Ministerio de Energía. Smart grids y la evolución de la red eléctrica. Informe publicado, 2011.

[13] J.M. Morales, R. Mínguez, and A.J. Conejo. A methodology to generate statistically dependent wind speed scenarios. Applied Energy, 87:843-855, 2010. 